HILPERT, KONRAD, Die Menschenrechte im Spiegel eines revolution?ren

Bildes , Zeitschrift f?r Religions- und Geistesgeschichte, 43 (1991) p.1

KONRAD HILPERT

\title{
Die Menschenrechte im Spiegel eines revolutionären Bildes*
}

\section{Die Beeindruckung der Augen und die Lenkung der Gedanken ${ }^{1}$}

Bilder und Bildsequenzen, gleich ob beiläufig protokollierend oder absichtsvoll inszeniert, spielen für das politische Bewußtsein des einzelnen wie für die Präsentation politischer Ereignisse und politischer Positionen durch Regierung, Parteien oder andere Akteure eine erhebliche Rolle. Nicht nur auf Fakten und Taten und auch nicht nur auf das gesprochene Wort kommt es an, sondern auch auf den Eindruck, den „Aussehen“ und „Darstellung“ hervorrufen. Jeder Wahlkampf mit seinen eigentümlichen Ritualen macht diesen Sachverhalt überdeutlich; doch ist das Visuelle auch sonst eine wichtige und kaum zu überschätzende Komponente des politischen Prozesses.

Die bildliche Umsetzung politischer Verhältnisse und Anschauungen, die heute vor allem von Photographien, Filmen, graphischem Layout, "Auftritten" vor der Öffentlichkeit besorgt wird, fiel in der Zeit vor der Erfindung der entsprechenden Techniken der Kunst zu. In der künstlerischen Darstellung des Bildes, der Skulptur und selbstverständlich auch der Architektur können politisch entscheidende Ereignisse der Mit- und Nachwelt erzählt werden. In der künstlerischen Darstellung können aber auch politische Gegebenheiten und Selbstverständnisse sinnenfällig faßlich gemacht werden, die sonst zu kompliziert, zu abstrakt oder nur in ihrer Pflichtseite erfahrbar wären. Schließlich können in Bildern auch Verbindungen hergestellt, Ansprüche transportiert und sogar normative Ideale in kritischer Absicht vergegenwärtigt werden.

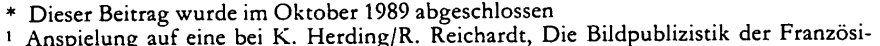
1 Anspielung auf eine bei K. Herding/R. Reichardt, Die Bildpublizistik der Franzosi-
schen Revolution, Frankfurt a. M. 1989, S. 15 f., zitierte Passage eines Leitartikels in der auflagenstärksten radikalen Zeitung aus dem Jahr 1792: „Despotismus und Aberglauben haben die Augen beeindruckt, um die Gedanken zu lenken und sich dienstbar zu machen; ein kundiger Gesetzgeber muß alles zu nutzen wissen. Dadurch, daß die Zeichen der Feudalität und der Monarchie beseitigt werden, verlieren sie sozusagen ihren Körper und hödalitä un lie übrigen dürfen die Blikren auf, die Sonne der Gewohnheitstiere gefangen zu neh. ke eines Volkes, das zur Republik erhoben wird, nur noch auf solche Gegensatze fallen, die sein Gemüt stärken und dauernd an die erhabenen Grundsätze der Gleichheit erinnern."
}

\section{Copyright (c) 2004 ProQuest Information and Learning Company Copyright (c) Brill Academic Publishers}


Die übers Erzählen hinausgehenden Funktionen der sinnenfälligen Darstellung und - wie wir heute wohl sagen würden - der Bewußtseinsbildung waren umso mehr auf das künstlerische Bild angewiesen, als andere Kommunikationsmittel, die ähnlich viele Menschen erreicht hätten, nicht vorhanden waren oder - wie die Schrift - nur von einer kleinen Elite benutzt werden konnten. In dieser Funktion des hauptsächlichen politisch-gesellschaftlichen Kommunikationsmediums hat die bildnerische Kunst in der abendländischen Kultur wie anderswo auch eine reiche Symbolwelt herausgebildet. Ihr Repertoire umfaßt sowohl Personifikationen abstrakter sozialer und moralischer Grundbegriffe als auch historische oder mythologische Gestalten, die bestimmte Verhaltensweisen oder Fähigkeiten typisch verkörpern. Dazu kommen vor allem seit dem Humanismus elaborierte Embleme, in denen Bildmotive aus Natur, menschlichem Alltag oder Vergangenheit mit kurzen Sentenzen zu festen Sinnzeichen verbunden sind. Gerade sie dürften sich aber meist nur Gebildeten, in manchen Fällen sogar nur speziell Eingeweihten, entschlüsselt haben, im Gegensatz zu den Allegorien und den typischen Gestalten, die nach einmaliger oder zyklisch wiederkehrender Interpretation wohl auch von einem größeren. Teil der einfacheren Leute verstanden worden sein dürften.

Symbole können einen Vorstellungsgehalt nur dann sichtbar machen, wenn sie in Form und Bedeutung nicht beliebig sind. Diese Nichtbeliebigkeit schließt aber keineswegs aus, daß jedem künstlerischen Bild, das Symbole verwendet, ein Moment von Rätselhaftigkeit eigen ist, das Interpretation nicht überflüssig sein läßt, sondern im Gegenteil herausfordert. Die künstlerische Kreativität und die je eigene Sicht des Künstlers ist nur ein Grund hierfür. Ein anderer liegt darin, daß sich Symbole immer wieder neu miteinander kombinieren lassen und daß - wie man schon im Mittelalter wußte - auch neue Aussagen mit Hilfe des Alten "offensichtlich" gemacht werden können. ${ }^{2}$ Schließlich liegt es an der Eigenart der Übertragung eines Begriffs und erst recht derjenigen eines komplexeren Vorstellungsgehalts selbst, daß das symbolhaltige künstlerische Bild nicht nur mitteilt oder schmückt, sondern auch affiziert und zu denken gibt: Die Übertragung bleibt stets assoziativ und die bildhaften Zeichen ungleich stärker als Begriffe und gar Texte offen für Erweiterungen, Verflachungen, Umdeutungen.

Bilder, die politische Ereignisse, Amtsträger oder auch grundsätzliche politisch-soziale Gegebenheiten und Probleme darstellen, kommentieren und mit Hilfe bereitliegender Zeichen deuten, sind also immer mehr als bloß Ausfluß subjektiver Sichten oder die realen Verhältnisse lediglich abbildende Scheinwelt. Vielmehr sind sie auch Faktoren und Instrumente im politischen Geschehen selbst. Ganz offensichtlich ist das dort, wo ein politisches Bild auf den Auftrag eines politischen Akteurs zurückgeht und der gesellschaftlichen Öffentlichkeit bzw. dem erreichbaren

\footnotetext{
2 Zum theoretischen und historischen Hintergrund s. einführend L. Kaute, Art. Allegorie, in: Lexikon der Christlichen Ikonographie I, S. 97-100.
}

\section{Copyright (c) 2004 ProQuest Information and Learning Company Copyright (c) Brill Academic Publishers}


Teil derselben zur Schau gegeben wird: Herrscherbild und kunstvolle Gestaltung von Bauwerken konnten deshalb über Jahrtausende ein (teilweise wohlbedachter) Bestandteil der Ausübung und ideellen Rechtfertigung politischer Herrschaft bilden ${ }^{3}$. Es trifft aber auch dort zu, wo Bilder mit politischen Sujets auf den Antrieb des einzelnen Künstlers selbst zurückgehen und nach ihrer Fertigung für jedermann sichtbar ausgestellt sind und erworben werden können ${ }^{4}$. Zumal wenn solche Bilder von vornherein oder auf dem Umweg des Kopierens in einer Technik ausgeführt wurden, die Vervielfältigung erlaubte, nahmen sie und die in ihnen enthaltene Botschaft am Prozeß der Formierung des politischen Bewußtseins und an der öffentlichen Meinungsbildung teil. Wieweit diese bildnerische Wirksamkeit reicht, hängt naheliegenderweise von einer ganzen Reihe von Faktoren ab: nicht nur von der ästhetischen Qualität und vom Interesse an den politischen Vorgängen, sondern auch von der Menge der Bilder und der Konsistenz ihrer Botschaften untereinander, von der Vertrautheit des Publikums mit den benutzten Symbolen, von den Sehgewohnheiten und nicht zuletzt vom Vorhandensein oder Nichtvorhandensein anderer Massenkommunikationsmittel, die bezüglich Eindringlichkeit, Aktualität, Multiplizierbarkeit und Mobilität mit den politischen Bildern konkurrieren konnten.

Von sämtlichen dieser Faktoren her fand die Druckgraphik im Frankreich der Revolutionszeit geradezu optimale Bedingungen vor: Es gab schon seit Jahrzehnten sowohl in Paris als auch in der Provinz ein blühendes Stecher- und Druckergewerbe samt einem entsprechenden Verlagswesen. Der Umgang mit Bildern und Bildblättern war auch den einfachen Leuten geläufig (Begegnung mit Bildern in Kirchen, an öffentlichen Gebäuden, aber auch in den Wohnungen [besonders religiöse Gebrauchsgraphik]). Die überwiegende Mehrheit der Bevölkerung konnte weder schreiben noch lesen ${ }^{5}$. Ein Medium, das bei der Vermittlung überlokaler Nachrichten und beim Austausch von Meinungen, Überzeugungen und Sichtweisen von Vorkommnissen, Besteuerung, militärischen Interventionen, drückenden Pflichten, Nöten, Belastungen und internen Spannungen, Lebensstil der Privilegierten usf. der Druckgraphik überlegen gewesen wäre, gab es noch nicht. Die Charakterisierung, die K. Herding und R. Reichardt für die Bildflugblätter geben,

3 Genaueres dazu (incl. Lit). s. etwa bei A. Reinle, Das stellvertretende Bildnis. Plastiken und Gemälde von der Antike bis ins 19. Jahrhundert, Zürich/München 1984, S. 66112 u. S. 250-319. M. Wanke, Das Bild als Herrschaftsbestätigung, in: Funkkolleg Kunst. Studienbegleitbrief 7, Weinheim/Basel 1985, S. 51-82. Eine exemplarische Detailstudie bietet: F. Matschke, Die Kunst im Dienst der Staatsidee Kaiser Karls VI. Ikonographie, Ikonologie und Programmatik des „Kaiserstils“, Berlin/New York 1981.

${ }_{4} \mathrm{Zu}$ den Herstellungs- und Absatzbedingungen der Druckgraphik z, Zt. der Franzö${ }^{4} \mathrm{Zu}$ den Herstellungs- und Absatzbedingungen der Druckgraphik z, Zt. der Franzo-
ischen Revolution s. u. a. J. Adhémar u. a., Populäre Druckgraphik Europas: Frankreich - vom 15. bis zum 20. Jahrhundert, München 1968, S. 90-94; Herding/Reichardt, Die Bildpublizistik (s. Anm. 1) S. 20-24.

5 F. Furet/J. Ozouf, Lire et écrire. L'alphabétisation des Français de Calvin à Jules Ferry, Bd. I, Paris 1977, geben den Anteil mit 2/3 an (nach Herding/Reichardt, Die Bildpublizistik [s. Anm. 1], S. 18-20).

\section{Copyright (c) 2004 ProQuest Information and Learning Company Copyright (c) Brill Academic Publishers}


läßt sich auf die Druckgraphik insgesamt ausweiten: Sie erfüllt „eine Schlüsselfunktion im damaligen politisch-sozialen Kommunikationssystem" "6. Daß sie nicht nur dokumentieren und illustrieren, sondern auch aktuell informieren, analysieren, ja deuten, aber auch entlarven und anklagen bzw. beeindrucken, appellieren, Einverständnis herbeiführen und sogar begeistern möchten, wird nicht bloß am genau geplanten Aufbau der Bilder und der Art der Darstellung (etwa Gestik und Blicke) deutlich, sondern vor allem an der starken „Aufladung" mit Symbolik. Scheint diese Codierung nicht auffällig genug oder die Mröglichkeit $\mathrm{zu}$ ihrer Interpretation zu vielfältig, so wird sie häufig durch Begriffe, ausführliche Bildunterschriften oder sogar Imperative, Fragen oder zitatähnliche Austufe ,vereindeutigt““.

Den Protagonisten der Revolution war die Bedeutung der politischen Bilder und Symbole für die politisch-soziale Kommunikation übrigens durchaus bewußt. So ist etwa im Leserbrief eines burgundischen Friedensrichters an eine revolutionäre Zeitung klarsichtig von einer „beredten Zeichensprache“ die Rede; er erläutert sie als Sprache, „die zunächst nur die Sinne anspricht, diese aber so erregt, daß der Eindruck weitergeleitet wird bis hin zum Zentrum der Gedanken; ein solcher Sinneseindruck ist gleichsam ein natürlicher, elektrischer Impuls, der das Herz neu belebt, mit einem heiligen Feuer erfüllt, das - wenn es erst einmal brennt - nicht wieder erlischt" "7. Konsequenterweise postuliert dieser Autor, die nationale (Um-) Erzichung habe mit einer revolutionären Zeichensprache zu beginnen!-

Im folgenden soll nun in mehreren Gängen versucht werden, am Beispiel einer auch in Deutschland sehr bekannten und häufig als schmükkende Illustration für die Menschenrechts-Idee verwendeten Druckgraphik den bildlichen Code ins Sprachliche zu übertragen. Die Darstellung, die vor allem in der von L. Laurent kopierten Fassung Verbreitung gefunden hat, stammt ursprünglich von J.-J.-F. Le Barbier ${ }^{8}$. Ihre genaue Entstehungszeit ist unbekannt, doch läßt sie sich eingrenzen auf den Zeitraum zwischen der auf der Überschriftplakette genannten Bestätigung durch den König (sie erfolgte am 5. 10.1789) und dem Fluchtversuch der königlichen Familie am 20. 6. 1791. Mit diesem Datum nämlich schlug die Stimmung endgültig gegen den König um; mit diesem Stimmungsumschlag, der sich in der Ablösung des Rufs „Vive le

${ }^{6}$ Herding/Reichardt, Die Bildpublizistik (s. Anm. 1), S. 18.

7 Zitiert bei Herding/Reichardt, Die Bildpublizistik (s. Anm. 1), S. 15. Die gleiche politisch-propagandistische Psychologie findet sich in den Beiträgen des Abbé H. Grégoire zur Frage des Staatssiegels der Republik; Auszüge daraus s. G. v. d. Heuvel, Der Freiheitsbegriff der Französischen Revolution. Studien zur Revolutionsideologie, Göttingen 1988 (= Schriftenreihe der Hist. Kommission bei der Bayer. Akademie der Wissenschaften 31), S. 184 f., und L. Hunt, Symbole der Macht - Macht der Symbole. Die Französische Revolution und der Entwurf einer politischen Kultur, Frankfurt 1989 (orig.: Politics, Culture, and Class in the French Revolution, University of California Press 1984), S. 112116.

${ }^{8}$ Näheres über diesen Künstler in; Nouvelle Biographie Générale depuis Les Temps les plus reculés Jusqu' à $1850-60, \mathrm{Bd}$. XXX, S. $63 \mathrm{f}$.

\section{Copyright (c) 2004 ProQuest Information and Learning Company Copyright (c) Brill Academic Publishers}


Die Menschenrechte im Spiegel eines revolutionären Bildes 5

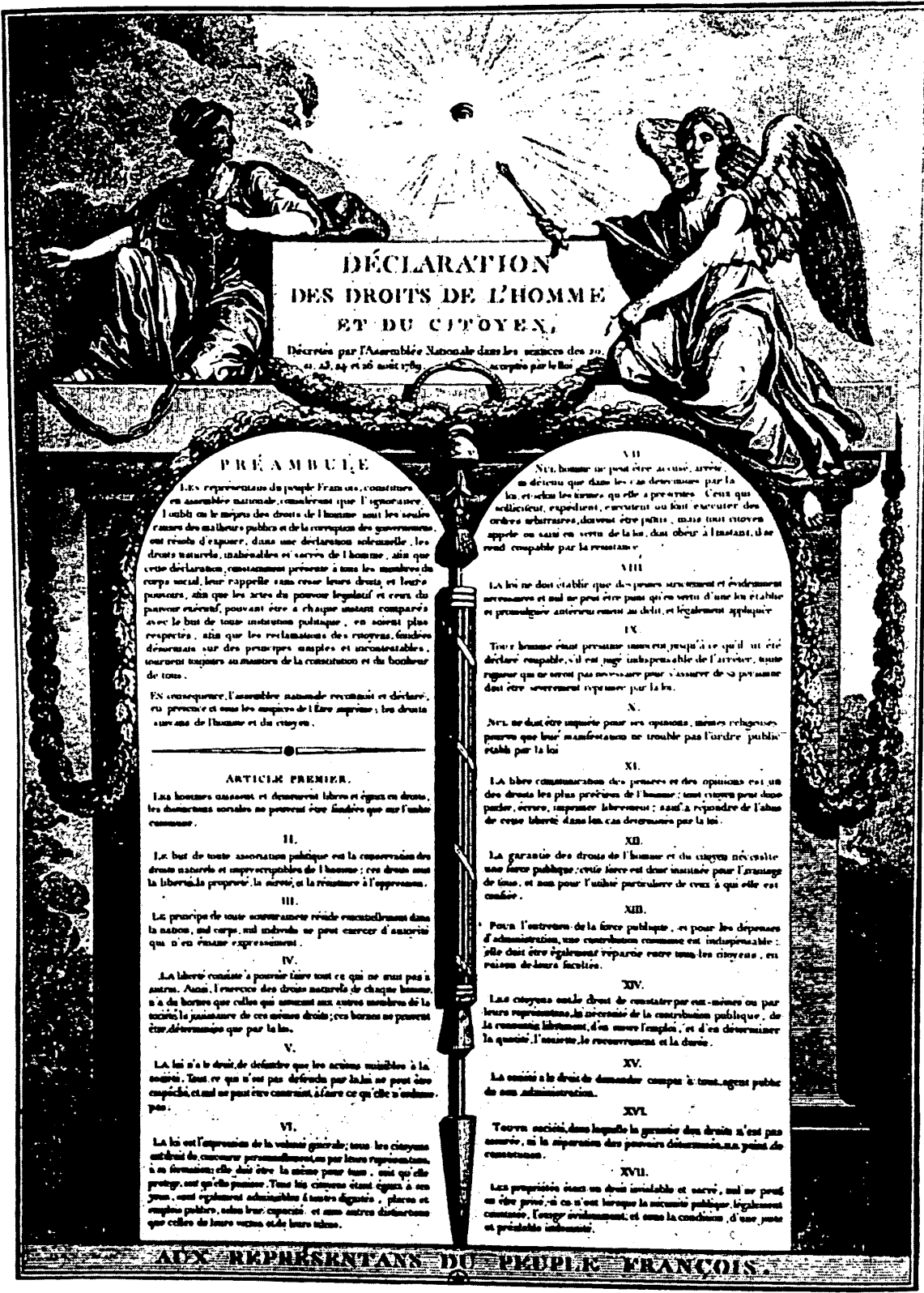

Quelle: K. Herding / R. Reichardt, Die Bildpublizistik der Französischen Revolution, Frankfurt 1989, S. 46 
Roi!“ durch „Vive la Nation!“, in der Beschädigung der Hoheitszeichen, im Auftauchen von Pamphleten und schließlich auch in der Forderung nach Absetzung äußerte 9 , war die (in unserem Bild durch die Königskrone auf dem Haupt der Allegorie der französischen Nation zum Ausdruck gebrachte) Überzeugung von der Versöhnbarkeit zwischen Revolution und Königtum endgültig dahin. Der König galt nun vor der Öffentlichkeit als erwiesener Konterrevolutionär, der gegenüber seinem Volke ein Doppelspiel getrieben hatte. Krone und Szepter als die traditionellen Insignien der Königsmacht tauchen in revolutionären Bildern nach diesem Datum nur noch in polemischer Brechung auf. Diese Datierung wird bestätigt durch den Wortlaut des französischen Textes; er bietet den 17. Artikel in der ursprünglichen pluralischen Fassung, die Anfang September 1791 in eine singularische verändert wurde.

Thema des Bildes ist die Menschenrechts-Deklaration vom 26. August 1789. Sprachliche und bildnerische Fassung werden hierbei eng miteinander verflochten. Anders als es die Nennung eines präzisen Datums eines Vorgangs und einer Widmung zunächst erwarten lassen könnten, handelt es sich nicht um ein erzählendes Bild im Sinne der Darstellung eines ereignishaften Augenblicks mit einem Vorher und Nachher oder eines szenischen Ablaufs. Vielmehr ist das Bild sehr statisch und wirkt geradezu lehrhaft. Die Erwartung, die es entstehen läßt, geht nicht in Richtung einer Schilderung von Episodischem, sondern in Richtung einer Erschließung eines feststehenden Sachverhalts.

\section{Komposition und emblematisches Inventar}

Das Bild ist streng aufgebaut. In der Horizontalen herrscht Achsensymmetrie vor, und zwat so stark, daß eben schon dadurch das Bild sehr statisch wirkt. In der Vertikalen gibt es eine Dynamik von unten nach oben, was vor allem durch die farbliche Abstufung von Dunkel nach Hell erreicht wird und in der kolorierten Fassung noch deutlicher ausfällt. In der Tiefe gliedert sich das Bild in drei Bereiche, nämlich in die Doppeltafel mit dem vollständigen Text der Menschenrechts-Deklaration von 1789, die Umrahmung in Gestalt eines allegoriengeschmückten öffentlichen Monuments sowie den Hintergrund, in dem naturalistisch dargestellter Raum und symbolisch codierte Transzendenz miteinander verknüpft sind.

Figürlich dominieren die beiden allegorischen Frauengestalten; ihre Gesten und Mienen bringen am ehesten Lebendigkeit ins Bild und möchten offensichtlich die Aufmerksamkeit des Betrachters leiten. Links (aus der Perspektive des Betrachters) - in der kolorierten Fassung in den Farben der Tricolore - die Verkörperung der französischen Nation. In den Händen hält sie die zerbrochenen Ketten. Im Begriff aufzustehen richtet sie ihren Blick erwartungsvoll oder konzentriert auf die

9 Das Umschlagen der Stimmung ist sehr anschaulich beschrieben bei E. Lever, Ludwig XVI., Stuttgart 1988 (orig.: Louis XVI, Librairie Arthème Fayard 1985), S. 440-476.

\section{Copyright (c) 2004 ProQuest Information and Learning Company Copyright (c) Brill Academic Publishers}


ihr herrscherlich gegenübersitzende (das Sitzen in Gegenwart anderer war ein Vorrecht!) Frauengestalt. Diese ist an den Flügeln, am Szepter ${ }^{10}$ und in der kolorierten Fassung auch an der rot-weißen Gewandung ${ }^{11}$ als Allegorie der Freiheit erkenntlich. Mit ihrer linken Hand verweist sie den Betrachter unmittelbar auf den Text der Menschenrechte. Mit dem Szepter, das sie in der rechten Hand hält und das hier kaum bloßes Herrschaftsinsignium ist, sondern anderen Darstellungen ${ }^{12}$ nach zu schließen auch die Vernunft symbolisieren könnte, verweist sie auf das Lichtdreieck mit Auge. So entsteht optisch eine sachlich bedeutsame Verbindungslinie Gott - Vernunft - Freiheit - Menschenrechte.

Zwischen den beiden allegorischen Gestalten, zugleich aber über ihnen ist am Himmel jenes Bildelement angeordnet, das sich am wenigsten bloß dekorativ oder bloß allegorisch ins Bildganze einfügt und sich damit am unmittelbarsten als Symbol zu erkennen gibt: eben das Lichtdreieck mit Auge. Als Zeichen für göttliche Wachsamkeit und übermenschliche Weisheit reicht die ikonographische Verwendung des Auges bis zu den Ägyptern zurück ${ }^{13}$. Im christlichen Einflußbereich wurde es seit dem 18. Jh. mit dem gleichseitigen Dreieck als Symbol der Trinität, das ebenfalls eine bis in die Antike zurückreichende, freilich nicht ungebrochene Tradition ${ }^{14}$ aufweisen kann, zu einem einzigen Sinnzeichen zusammengefaßt. Zur Zeit der Aufklärung ist der Hinweis des Zeichens auf den christlichen Gott aber nicht mehr eindeutig: Das ins Dreieck eingeschriebene Auge steht auch für Erleuchtung, tiefere Einsicht, un-

\footnotetext{
10 Im Gegensatz zu den Flügeln, die nur auf manchen Darstellungen der Liberté zu sehen sind (Beispiele etwa bei Heuvel, Der Freiheitsbegriff [s. Anm. 7], S. 185, und Herding/Reichhardt, Die Bildpublizistik [s. Anm. 1], S. 34 f.), erscheint das Szepter als ein festes (später durch die Pike ersetztes) Attribut der Liberté. Das für die Bildwelt der Französischen Revolution wohl bedeutsamste ikonologische Musterbuch von H. F. Gravelot/C. N. Cochin (Iconologie ou Traité de la Science des Allegories à l'usage des Artistes en 350 Figures, 4 Bde., Paris 1791) nennt es ganz ausdrücklich (III, S. 31). Zur ikonographischen Darstellung der Liberté in der Französischen Revolution s. außerdem M. Agulhon, Marianne au Combat. L'imagerie et la symbolique républicaines de 1789 à 1880 , Paris 1979 , S. 21-53, und Heuvel, Der Freiheitsbegriff [s. Anm. 7], S. 167-192. 11 Vgl. den Hinweis bei J. P. Bertaud, Alltagsleben während der Französischen Revolu-
ion, Freiburg/Würzburg 1989 (orig.: La vie quotidienne en France au Temps de la Révolution 1789-1795, Paris 1983), S. 89.

12 Ein prominentes Beispiel ist die Vignette auf dem Titelblatt des ersten Bandes der

Encyclopédie aus dem Jahr 1751 .
13 Zur Geschichte des Augensymbols s. u. a.: D. Forstner, Die Welt der christlichen 13 Zur Geschichte des Augensymbols s. u. a.: D. Forstner, Die Welt der christlichen
Symbole, Innsbruck u. a. 1977, S. 331-333; D. de Chapeaurouge, Einführung in die Geschichte der Christlichen Symbole, Darmstadt 1984, S. 145 f.; Herding/Reichardt, Die Bildpublizistik (s. Anm. 1), S. 26 u. S. 44-49. Einen originellen Uberblick über die weitere Geschichte des Augensymbols bis in unsere Gegenwart bietet A. Stock, Der göttliche Augenblick, in: $\mathrm{Ch}$. W. Thomsen/H. Holländer (Hg.), Augenblick und Zeitpunkt. Studien zur Zeitstruktur und Zeitmetaphorik in Kunst und Wissenschaften, Darmstadt 1984, S. 208-221.

14 Als Trinitätssymbol wurde das Dreieck offensichtlich zum ersten Mal von den Manichäern benutzt. Augustinus verwarf es (In Ev. Joannis CXXII/8) und legte damit die Nichtverwendung dieses Symbols bis ins 17. Jahrhundert hinein fest (nach: Chapeaurouge, Einführung [s. Anm. 13], S. 111 f.)
}

\section{Copyright (c) 2004 ProQuest Information and Learning Company Copyright (c) Brill Academic Publishers}


bestechliche Wahrnehmung. ${ }^{15}$ Im vorliegenden Bild ergibt sich die nähere Bedeutung aus dem zweiten Absatz der Präambel, in dem die Erkenntnis und die öffentliche Bekanntmachung der Menschenrechte auch wortmäßig ,in [der] Gegenwart und unter dem Schutze des Höchsten Wesens " verortet werden. Das Element einer höheren Legitimation ${ }^{16}$ ist hierin ebenso impliziert wie das Element der Wachsamkeit und das der erkenntnismäßigen Herkunft. Letzteres ist vielleicht sogar besonders dominierend, wenn man die vom Dreieck als einziger Lichtquelle des Bildes ausgehende Strahlung und den im Hintergrund sichtbaren Kontrast zu den drohenden Wolken und der undurchsichtigen Dunkelheit (besonders rechts unten) berücksichtigt. Vielleicht stehen diese sogar als Bilder für die Vergangenheit, deren Dunkel, Trübheit und Gewölk durch das strahlende Licht vertrieben werden. Wahrscheinlich reicht die symbolische Bedeutung des Lichtdreiecks in unserem Bild aber noch weiter: Die Gleichseitigkeit des Dreiecks signalisierte dann die Gleichrangigkeit der drei Stände. Dafür spricht vor allem, daß das Dreieck in vielen Bildern aus der Revolutionszeit als Attribut der allegorisierten Liberté vorkommt, ${ }^{17}$ nicht selten sogar zusammen mit denselben Symbolen, die auf unserem Bild in die monumentale Umrandung der beiden Menschenrechts-Tafeln integriert sind. Die Gleichrangigkeit der göttlichen Personen im einen Gott würde auf diese Weise zum Sinn- und Urbild der Gleichrangigkeit der Stände im einen Volk.

Als Trennachse zwischen den beiden Texttafeln fungieren eine Reihe von Gegenständen, die zum Standardrepertoire revolutionärer Darstellungen gehören: Pike mit Liktorenbündel, Freiheitsmütze und ringförmige Schlange.

Die Pike war eine Waffe, die zum einen leicht herzustellen war, zum anderen im Unterschied zu Säbel, Degen, Gewehr und Pistole auch ohne besonderes Training wirkungsvoll gehandhabt werden konnte. Sie war deshalb in militärischen Kreisen verpönt. Als Waffe des einfachen Volkes spielte sie aber eine wichtige Rolle in den vielen gewaltsamen Auseinandersetzungen zwischen alter Ordnung und revolutionärer Gegenmacht. So gewann sie schnell symbolische Bedeutung als Zeichen für die Volkssouveränität (und wurde übrigens auch schon bald zum Teil der Standardausrüstung der französischen Truppen ${ }^{18}$ ). In zahlreichen Dar-

$15 \mathrm{Vgl}$. Herding/Reichardt, Die Bildpublizistik (s. Anm. 1), S. 44 f.

16 Jahrhundertelang begannen königliche Urkunden mit der Formel „Im Namen der heiligen und unteilbaren Dreifaltigkeit". - Zur Debatte über diesen Punkt bei der Beratung der Menschenrechts-Erklärung s. etwa B. Plongeron, Die Geburt einer republikanischen Christenheit (1789-1801): Abbé Grégoire, in: Concilium (D) 25 (1989), S. 19-28, hier: S. $22 \mathrm{f}$.

17 S. dazu die Deutung des Gemäldes von Regnault bei Chapeaurouge, Einführung (s. Anm. 13), S. 112. Zur facettenreichen Entwicklung und Transformation des Dreieck Symbols in der Revolutionszeit s. J.A. Leith, Les étranges Métamorphoses du Triangle pendant la Révolution française, in: M. Vovelle (Hg.), Les Images de la Révolution Française. Actes du colloque du 25.-27. 10. 1985 tenu en Sorbonne, Paris 1988 (= Histoire moderne 21), S. 251-259.

$18 \mathrm{Vgl}$. Bertaud, Alltagsleben (s. Anm. 11), S. 52 f.

\section{Copyright (c) 2004 ProQuest Information and Learning Company Copyright (c) Brill Academic Publishers}


stellungen aus der republikanischen Phase der Revolution erscheint sie in der Hand der Liberté. In unserem Bild wird diese Zeichenhaftigkeit noch bekräftigt, indem die Pike durch ein Liktorenbündel hindurchgesteckt ist, dem Zeichen der Amts- und Strafgewalt hoher Beamter in Rom $^{19}$. Zugleich sah man in den zusammengebundenen Ruten schon seit dem 17. Jahrhundert ein Symbol der Einheit und Eintracht, das man in der republikanischen Phase auf die unteilbare Einheit der 83 Départements applizierte 20.

Dieses Symbol-Ensemble wiederum trägt die Freiheitsmütze, die in den Dörfern und Städten, aber auch häufig in der bildlichen Darstellung den Freiheitsbaum krönt. Die Freiheitsmütze wurde im antiken Rom als Zeichen verwendet, mit dem der Herr seinen Sklaven in den Status eines Freigelassenen überführte (pileus). Bereits in der Antike wurde sie in der charakteristischen Gestalt der Kopfbedeckung eines kleinasiatischen Seeräubervolkes („phrygische Mütze") dargestellt. In allen republikanischen Bewegungen der Neuzeit gewann sie die Bedeutung eines Symbols für Befreiung aus der Knechtschaft. Im revolutionären Frankreich wurde sie vor allem seit der Entlassung der Galeerensträflinge von Brest 1792, deren traditionelle Kopfbedeckung sie gewesen war, populär. Entschiedene Verfechter der Republik, vor allem aus dem Umkreis der Jakobiner (deshalb ja auch "Jakobinermütze“ genannt), machten sie zu einem der wichtigsten Abzeichen der politischen Gesinnung des einzelnen in der Öffentlichkeit, darüber hinaus aber auch zu einem praktisch allgegenwärtigen Requisit der nationalen Selbstdarstellung auf Siegeln, Poststempeln, Armeefahnen u. ä. ${ }^{21}$.

Im Schnittfeld der weit ausladenden, die Bedeutung der beiden Texttafeln hervorhebenden Girlanden - bezeichnenderweise aus Eichenlaub und Eicheln (als Freiheitsbäume wurden stets junge Eichen verwen$\operatorname{det} !^{22}$ ) - befindet sich die Schlange. Diese verschlingt ihren Schwanz und bildet so einen Ring. Es handelt sich hierbei nicht um das aus der christlichen Ikonographie geläufige Teufelssymbol, sondern um die Uroboros-Schlange, die im hellenistischen Kultursynkretismus eine Rolle spielte und seit den griechischen Texteditionen im Zeitalter des Humanismus an Bedeutung gewonnen hat. Mit ihrer Kreisgestalt symbolisiert sie die Unsterblichkeit, weshalb man ihr häufig gerade auch auf

19 Näheres in: Der Kleine Pauly. Lexikon der Antike, Stuttgart 1967-1975, III, S. 645 f.: Art. Lictor

20 S. A. Mathiez, Les Origines des Cultes Révolutionnaires 1789-1792, Paris 1904, S. 34; Chapeaurouge, Einführung (s. Anm. 13), S. 120 f.; Hunt, Symbole der Macht (s. Anm. 7), S. 80 u. S. 122.

${ }_{21}$ Weiteres dazu in A. Rabbow, dtv-Lexikon politischer Symbole A-Z, München 1970 , S. 34; Chapeurouge, Einführung (s. Anm. 13), S. 144 f.: Heuvel, Der Freiheitsbegriff (s. Anm. 7), S. 192-198; E. Liris, Autour des Vignettes Révolutionnaires: La Symbolique du Bonnet Phrygien, in: Vovelle (Hg.), Les Images (s. Anm. 17), S. 307-315.

22 S. Rabbow, dtv-Lexikon (s. Anm. 21), S. 91. Vg1. Mathiez, Les Origines (S. Anm. 20), S. 32 f..

\section{Copyright (c) 2004 ProQuest Information and Learning Company Copyright (c) Brill Academic Publishers}


Sepulkralmonumenten begegnet ${ }^{23}$. Als symbolisch bedeutsam erschließt sich dem kundigen Betrachter freilich auch das Ineinander von sich verjüngendem Schwanz und ausgewachsenem Kopfteil sowie der Vorgang der Häutung 24: Altes wird abgelegt, weil es nicht mehr paßt; hervor geht aus diesem Prozeß aber nicht ein reduziertes Altes, sondern das verjüngte, vitale Bleibende.

\section{Schauen und Lesen}

Bereits bei einem flüchtigen Blick fällt an unserer Darstellung die Verbindung von Bild und Text auf. Innerhalb der Druckgraphik ist das freilich weder von der Tradition her noch im Kontext der revolutionären Bilder insgesamt etwas Besonderes. Besonders sind in diesem Fall freilich Anteil und Art des Textes: Die Darstellung beläßt es nämlich nicht, wie sonst bei einer Inschrift, die für ein ganzes Korpus steht, bei einer Widmung und einer zentralen Aussage, sondern zitiert den gesamten Text der Menschenrechts-Erklärung. Der Anteil, der dafür von der gesamten Bildfläche beansprucht wird, ist ungewöhnlich groß. Handelt es sich also eher um ein dekoratives Textblatt als um ein Bild mit einer ausführlichen sprachlichen Legende?

Bei genauerem Betrachten zeigt sich, daß die Beziehungen zwischen bildlichen und sprachlichen Anteilen mehrschichtiger sind, als es diese zunächst naheliegende und hilfreiche Alternative zuzulassen scheint. Es ist vor allem der Habitus der Liberté, der das Entweder-Oder stört: Ihr Blick fängt den Blick des Betrachters unwillkürlich auf; sie ist es aber auch, die ihn mit unmißverständlichem, man möchte fast sagen: keinen Widerspruch duldenden Gestus auf den Text der Deklaration verweist. Ein bildliches Element fungiert hier also als Anleitung zum Lesen eines Textes, also im eigentlichen Sinn als „Legende“. Ohne Bild wäre der Text bloß Zitat oder Dokument.

Diese appellative Relation, die das Bild gleichsam aus der Ebene des Bildes heraus zum Text hin aufbaut, ist freilich nur eine der Verbindungen zwischen Visuellem und Sprachlichem. Weitere bestehen im Binnenbereich der Darstellung selbst. So wird ebenfalls über die Gestik der bildnerischen Hauptfigur und hervorgehoben durch das in der rechten Hand geführte Szepter wie schon erwähnt eine Verbindung zwischen dem Strahlendreieck mit Auge und dem Text betont. Die dort aufgeführten Rechte werden dadurch als ganz besondere qualifiziert: Es sind nicht irgendwelche Rechte neben anderen, zwar verbindlich, aber gesetzt, abänderbar und aufhebbar, von Menschen (wenn auch besonders

${ }^{23}$ Ein schönes Beispiel bietet der Epitaph des Konstanzer Fürstbischofs Karl Theodor von Dalberg, abgebildet in: E.L. Kuhn u. a. (Hg.), Die Bischöfe von Konstanz, 2 Bde., Friedrichshafen 1988, Bd. II, S. 119.

${ }^{24}$ Chapeaurouge, Einführung (s. Anm. 13), S. 85, faßt die im Horapollo (eine nur in griechischer Ubersetzung erhaltene Schrift über Hieroglyphen aus dem 5. Jh.) gegebene Deutung folgendermaßen zusammen: „So wie die Schlange jährlich ihre Haut abstreife, um sich zu verjüngen, bewirke der Kreislauf des Jahres die Verjüngung der Welt.““

\section{Copyright (c) 2004 ProQuest Information and Learning Company Copyright (c) Brill Academic Publishers}


mächtigen) erfunden und betreut, sondern „die natürlichen, unveräuBerlichen und geheiligten Menschenrechte "25. Das Höchste Wesen bzw. Gott erscheint als ihr letzter Grund und schöpferischer Ursprung, zudem aber auch als erleuchtender erkenntnismäßiger Beistand und als wachsamer Garant ihrer öffentlichen Bekanntmachung (,en présence et sous les auspices de l'Être suprême" ${ }^{26}$ ). Nicht nur der Inhalt des Textes, sondern auch die Erklärung selbst erhalten hier eindrucksvoller als in den leicht überlesenen Einleitungsworten des Textes eine Legitimation "von oben": Die Menschenrechte erscheinen in der ungebrochenen Linie Gott-Vernunft-Freiheit-Menschenrechte als Manifestation göttlicher Vernunft und göttlicher Vor"sehung". Insofern erfüllt hier das Bild gegenüber dem Text eine akzentuierende Funktion.

Selbstverständlich gibt es auch in dieser Darstellung (wie in allen Bild/Text-Darstellungen dieser Zeit) Bezüge zwischen Bild und Text, die man als iterativ charakterisieren könnte. Das meint, daß im Bild ein (oder mehrere) Element(e) des Textes visuell wiederholt und zugleich veranschaulicht wird bzw. das Bild oder ein Bildteil durch Worte gefaßt und erklärt wird. So entspricht die Formation von Wolken und freiem Himmel und das Spiel zwischen Dunkel und Hell dem Hinweis auf „das öffentliche Unglück und die Verderbtheit der Regierungen“, auf „die Unkenntnis, das Vergessen und die Mißachtung der Menschenrechte" 27. Die zerbrochenen Ketten in den Händen der France korrespondieren mit der im zweiten Artikel erwähnten Unterdrückung. Das Liktorenbündel mit der Pike als Horizontalachse des Monuments verbildlicht den Grundsatz der Volkssouveränität in seinem in den Artikeln 3 und 12 jeweils positiv wie negativ formulierten ausnahmslosen Anspruch. Aber auch in umgekehrter Richtung ist das Bild in seiner Symbolik und Programmatik auf den Text innerhalb des Bildes angewiesen: Ohne Inschrift auf der Tafel zwischen den beiden allegorischen Figuren und ohne Deklarationstext wäre es zwar nicht unverständlich, aber mehrdeutig. Fehlte aber nur der Text der einzelnen Artikel, bliebe die Botschaft von den gottgewollten und allen sozialen Unterschieden vorausgehenden und unveräußerlichen Rechten des Menschen qua Mensch, wie sie besonders in der Präambel und in den Artikeln 1, 4, 5 und 16 herausgestellt wird, ein glänzendes, aber diffuses Ideal.

So wie der vollständige Text der Menschenrechte das Ideal der Freiheit expliziert und für den einzelnen Betrachter bzw. Leser unmittelbar handgreiflich macht, erfüllt umgekehrt die bildliche Darstellung gegenüber dem Text eine konzentrierende Funktion: Ähnlich wie die zusammenfassende Benennung als „die Rechte des Menschen und Bürgers“ in Präambel und abschließendem Artikel 17, läßt die bildliche Darstellung Freiheit, französische Nation, Volkssouveränität und Höchstes Wesen

25 Préambule. (Eine deutsche Übersetzung der Menschenrechts-Erklärung von 1789 findet sich etwa in: W. Heidelmeyer [Hg.], Die Menschenrechte. Erklärungen, Verfassungen, Internationale Abkommen, Paderborn 1972, S. 56-59).

26 Ebd.

27 Ebd.

\section{Copyright (c) 2004 ProQuest Information and Learning Company Copyright (c) Brill Academic Publishers}


als die zentralen Bezugsgrößen für Idee, Postulat und Realisierung der Menschenrechte erscheinen. Die Bedeutung solcher Verortung der Menschenrechte auf einer zweiten, wesentlich allgemeineren und abstrakteren Denkebene darf nicht unterschätzt werden: Sie läßt nämlich das in den einzelnen Artikeln Ausgeführte als Gesamtheit oder als situationsbedingt besonders dringliches Einzelrecht auch da gegenwärtig sein, wo die textliche Fassung nicht gelesen werden kann, weil entsprechende Fertigkeiten fehlen (etwa bei Kindern und des Lesens unkundigen Erwachsenen), eine Verlesung nicht vorgesehen ist (bei zahllosen staatlichen Akten) oder als überflüssig erschiene (weil man sie z. B. als Inhaber eines öffentlichen Amtes oder als interessierter Bürger schon einmal zur Kenntnis genommen hat). Die zusätzliche Umschreibung vieler detaillierter Postulate mit wenigen, dafür äußerst allgemeinen Bezugsgrößen liegt nicht nur im Interesse der inneren Systematisierung, sondern erhöht, zumal wenn sie auch noch visuell leicht zugänglich ist, die Redundanz der Detailaussagen. Die aber ist eine wesentliche Bedingung für bewußtseinsmäßige Aneignung und fortdauernde gesellschaftliche Relevanz.

\section{Kontinuitäten, Aktualisierungen, Brüche}

Unsere Menschenrechts-Darstellung enthält nicht nur wie jedes Bild eine implizite zeitgeschichtliche Dimensionierung, sondern sie führt auf der Inschrifttafel die Daten der fünf Sitzungstage auf, an denen die (in den vorausgegangenen Wochen aus einer Versammlung der Generalstände ausgegliederte und zur Repräsentantin der Gesamtnation umfunktionierte, nach dem Mehrheitsprinzip beschließende) Nationalversammlung die Menschen- und Bürgerrechte verhandelte, beschloß und zu einer feierlichen Erklärung zusammenfaßte. ${ }^{28}$ Trotz dieser präzisen Datierung verbildlicht unsere Darstellung aber nicht eigentlich ein punktuelles Ereignis der Vergangenheit, wie es bei anderen politischen Bildern aus dieser Zeit der Fall ist, die beispielsweise die Versammlung der Generalstände, die Erstürmung der Bastille oder später die Vereitelung der Flucht der Königsfamilie darstellen. Die Datierung muß vielmehr im Zusammenhang mit den anderen Zeit,,angaben" im Bild gesehen und von ihnen her verstanden werden: dem Uroboros-Emblem und der kompositorischen Gestaltung des gesamten Bildes als Denkmal. Das Schlangen-Symbol für Unsterblichkeit und Regeneration ist denkbar zentral und in größtmöglicher Nähe zur Datierung angebracht. Ein Denkmal aber ist nie bloß eine Würdigung der Verdienste der in der Widmung Genannten durch die Späteren, sondern es möchte immer auch und gerade bestimmte Ereignisse oder Haltungen und Handlungs-

28 Den genauen Ablauf der Beratungen und der Verabschiedung schildert S.-J. Samwer, Die französische Erklärung der Menschen- und Bürgerrechte von 1789/91, Kiel o. J. (=Veröffentlichungen des Instituts f. Internation. Recht. a. d. Univ. Kiel 63), S. 98-228. Zur vorausgegangenen parlamentarischen Revolution s. zusammenfassend E. Schulin, Die französische Revolution, München 1988, S. 61-64.

\section{Copyright (c) 2004 ProQuest Information and Learning Company Copyright (c) Brill Academic Publishers}


weisen von Personen als für die Gegenwart gültig und für die Zukunft verpflichtend festhalten.

Dies nun entspricht ganz den zeitlichen Bestimmungen, die im Text der Präambel für die Menschenrechte urgiert werden: Es handle sich um "natürliche" Rechte, die zwar nicht gekannt, vergessen oder mißachtet werden können, aber niemals abgeschafft werden können. Ihre feierliche Deklaration zielte darauf, sie "ständig gegenwärtig" zu halten und „ohne Unterlaß“ an sie zu „erinnern“! Es sollen nicht neue Rechte statuiert und proklamiert werden, sondern lediglich diejenigen in Worte gefaßt und öffentlich bekanntgemacht werden, die schon immer in Geltung sind (dies ist auch durch die juristische Qualifizierung als „Déclaration“" mitgesagt ${ }^{29}$ ); ferner soll die Entschlossenheit bekräftigt werden, daß sie von jetzt an nicht mehr vergessen oder mißachtet werden.

Von daher gesehen vermittelt die Datierung auf unserem Bild zwar auch eine historische Nachricht, in erster Linie jedoch markiert sie eine Zäsur: Zur Vergangenheit hin wird die Erklärung der Menschenrechte als Vergewisserung und öffentliche Bekanntmachung immer und ,von Natur" gültiger Rechte begriffen, also als Durchbruch und Erfüllung, wenn man so will. Das Neue aber, das alle Geschichte nach der Zukunft hin grundlegend verändern soll, besteht darin, vom selben Datum an Unkenntnis, Vergessen und Mißachtung dieser Rechte nicht mehr zuzulassen.

Diese doppelte Botschaft „Wiedereinsetzung des schon immer Gültigen" und andererseits „entscheidender Neubeginn" hat auch im Bild weitere - teils ausdrückliche, teils nur indirekt erschließbare - Entsprechungen.

So greift das Bildmaterial sowohl in den Symbolen als auch in Kleidung und Architektur auf antike, besonders auf römische Elemente zurück und lehnt sich merklich an sie an. Der Gesamteindruck ruft Marxens bissige Worte ins Gedächtnis, die Französische Revolution habe sich als römische Republik (und später als römisches Kaisertum) „drapiert“, „um in dieser altehrwürdigen Verkleidung und mit dieser er-

29 Näheres zu der literarischen Gattung der Deklaration bei Y. M.-J. Congar, Was besagt die Bezeichnung „Erklärung"?, in J. Hamer/Y. Congar (Hg.), Die Konzilserklärung über die Religionsfreiheit, Paderborn 1967 (= Konfessionskundlich u. kontroverstheologische Studien 20), S. 53-58.

\section{Copyright (c) 2004 ProQuest Information and Learning Company Copyright (c) Brill Academic Publishers}


borgten Sprache die neue Weltgeschichtsszene aufzuführen"30. In diesem antikisierenden Timbre drückt sich die Überzeugung aus, Freiheit des Bürgers und Ordnung des Staates hätten in Griechenland und Rom eine ideale Synthese gefunden, besonders in den republikanischen Epochen. Diese Überzeugung und Bewunderung war keine Erfindung der Revolutionäre, sondern aus der politischen Philosophie vor 1789 durchaus bekannt, ähnlich wie auch einzelne bildnerische Formen, die in unserem Bild verwendet werden, schon wenigstens seit der Jahrhundertmitte und z. T. weit darüber hinaus bekannt waren. Neu war jetzt, daß ganze Bilder aus ihnen aufgebaut wurden und vor allem, daß die Symbole und Allegorien eine geradezu kanonische Konsistenz bekamen. Viel entscheidender war freilich, daß solche Bilder an die Stelle anderer Bilder traten. $\mathrm{Ob}$ dies vom Künstler intendiert war oder ob hier Moden und verschüttete Traditionen wirkten, läßt sich im Einzelfall nur schwer sagen. Fest steht aber, daß der Austausch von Bildern und Symbolen in der revolutionären Propaganda insgesamt eine wichtige Rolle gespielt hat und ein Großteil der Betrachter die revolutionären Bilder so lesen mußte.

Solches An-die-Stelle-treten kann auf recht verschiedene Weise erfolgen. Die drastischste ist sicherlich die ausdrückliche, polemische Distanzierung von den Symbolen von gestern. Dieser Weg ist in unserem Bild nicht eingeschlagen. Es setzt sich zwar ebenfalls deutlich von der Vergangenheit ab, indem es Wolken, Dunkel und Ketten als Metaphern für die frühere Epoche wählt, aber es zerbricht nirgendwo deren Symbole (etwa die königlichen Insignien, die nach Abschaffung der Monarchie am 10. 8. 1792 auch real zerbrochen und zu republikanischen Münzen umgeschmolzen wurden ${ }^{31}$ ) oder verspottet sie auch nur.

Freilich gibt es auch sublimere Weisen des An-die-Stelle-Tretens wie die Besetzung und die Ablösung. Beide spielen sich weniger oder überhaupt nicht auf der Ebene reflexer Wahrnehmung ab. Sie können deshalb einerseits sehr nachdrücklich wirken; andererseits spielt sich diese Wirksamkeit jedoch im Verborgenen ab und ist deshalb im Ergebnis kaum zu ermessen, geschweige denn gezielt zu steuern. Mit Besetzung ist der Vorgang einer Umkodierung gemeint, was soviel heißt wie, daß ein

${ }^{30}$ K. Marx, Der achtzehnte Brumarie des Louis Bonaparte (1852), in: ders./F. Engels, Gesamtausgabe (MEGA), I/2, Berlin 1985, S. 97. Dabei handelt es sich nicht einfach um eine beschreibende Feststellung, sondern um eine analytische Aussage über das Wesen bürgerlicher Revolutionen, die etwa zu dem Satz zusammengefaßt wird: „Die früheren Revolutionen bedurften der weltgeschichtlichen Rückerinnerungen, um sich über ihren eigenen Inhalt zu betäuben." (ebd. S. 101, vgl. S. 96-102). Zum historischen Einfluß antiken Gedankenguts auf die Ausformulierung der Menschenrechte s. H. Cancik, Gleicheit und Freiheit. Die antiken Grundlagen der Menschenrechte, in: G. Kehrer (Hg.), „Vor Gott sind alle gleich". Soziale Gleichheit, soziale Ungleichheit und die Religionen, Düsseldorf 1983, S. 190-211. Zum systematischen Problem des Neubeginns und der Verwendung von Tradition in diesem Zusammenhang s. H. Arendt, Ubber die Revolution, München 31986 (orig.: On Revolution, New York 1963), S. 23-33, S. 63-72, S. 270-276, S. 277-362; J.-M. Domenach, Revolution und Moderne, in: Merkur 43 (1989) S. 1-12.

31 Nach Hunt, Symbole der Macht (s. Anm. 7), S. 114. 
vertrautes Bildmotiv oder Symbol mit einem veränderten Inhalt verbunden wird. So dürfte damals jeder, ob jung oder alt, reich oder arm, gebildet oder Analphabet, die Doppeltafel von der christlichen Darstellung der Offenbarung der Zehn Worte an Mose auf dem Berg Sinai gekannt haben.

Nach Ex 34, 28f. und Dtn 4,13 (vgl. Dtn 10, 1-5) schrieb Mose die Zehn Worte auf zwei steinerne Tafeln und brachte sie so dem Volk. Es handelt sich dabei um eines der ältesten Bildmotive der christlichen Kunst ${ }^{32}$. Vor allem seit dem Spätmittelalter galt der Dekalog nicht nur in der Theologie als wichtigstes Einteilungsprinzip der Pflichtenlehre ${ }^{33}$, sondern auch in der kirchlichen Volksunterweisung als Zusammenfassung der christlichen Moral ${ }^{34}$. In Adaption von Dtn 6, 4-9 gehörte die Doppeltafel mit dem Dekalog zum bildnerischen Standardrepertoire zahlreicher Kirchen.

Der Künstler unseres Bildes übernimmt dieses Schema und auch die Numerierung der Artikel mit den darübergesetzten römischen Zahlen, obschon der verabschiedete Text selbst die zugehörige Ordnungszahl in arabischen Ziffern schreibt und in den Text einrückt $t^{35}$.

Diese Übernahme ist umso bemerkenswerter, als die Aufteilung der Artikel auf zwei Tafeln im Gegensatz zur traditionellen Ordnung der Gebote keine inhaltliche Zusammengehörigkeit erkennen läßt. Indem er die Menschenrechte in dieses äußerst populäre, didaktisch prominente und moralisch mit höchster Verbindlichkeit versehene Schema einschreibt, werden sie als Grundlage des Zusammenlebens im Staat und gleichzeitig als Konzentrat der Moral des Bürgers präsentiert und konnotiert. Dabei muß sich das Neue dieses Arragements gar nicht auf die inhaltliche Neuheit der Grundsätze richten. Vielmehr geht es - jedenfalls in erster Linie - darum, durch die Übernahme und Weiterführung eines erfolgreichen Musters kirchlicher Moraldidaktik ein Bewußtsein der Menschenrechte in der Alltagswelt zu schaffen. Der Ort, für den ein solches Bild gedacht war, war kaum die Kirche oder der Glaubenskatechismus, sondern die öffentlichen Gebäude und all jene Stellen, wo der einzelne das Handeln des Staates konkret erfuhr: beim Unterricht in der Schule, bei der Gerichtsverhandlung, bei der Beurkundung von Verträgen, vor dem Standesamt, bei der Ausbildung zum Militärdienst, in Versammlungsräumen. ${ }^{36}$

\footnotetext{
32 Nach G. Schiller, Ikonographie der christlichen Kunst, Bd. IV./1, Gütersloh 1976, S. $121-123$

33 Für seine zentrale Stellung war vor allem die Autorität des Augustinus maßgebend.

34 Zur Bedeutung des Dekalogs in der christlichen Uberlieferung s. etwa R. Hofmann, Die Bedeutung des Dekalogs, theologisch und geschichtlich, in der Sicht der katholischen Moraltheologie, in: Zeitschr. f. Evang. Ethik 13 (1969), S. 230-245.

35 Den französischen Wortlaut bietet Samwer, Die französische Erklärung (s. Anm 27), S. 222-225.

${ }_{36} \mathrm{Vgl}$. hiermit die Charakterisierung der Funktion der Druckgraphik seit dem Spätmittelalter „als Propagandainstrument, ja als sinnliche Instanz verborgener, höherer Gewalt oder abstrakter Glaubenswahrheiten“ und als „von allen gefordertes Idealbild“, das „ein Bewußtsein gemeinsamer Interessen schuf", bei Herding/Reichardt, Die Bildpublizistik (s. Anm. 1), S. 25
}

\section{Copyright (c) 2004 ProQuest Information and Learning Company Copyright (c) Brill Academic Publishers}


Etwas gewagter ist die Vermutung, daß auch die Anordnung der oberen Bildhälfte und das Spiel des Lichts einen herkömmlichen und sehr verbreiteten Bildtyp der christlichen Ikonographie aufnimmt und umbesetzt, nämlich den Typus des Verkündigung-an-Maria-Bildes (auf der Grundlage von Lk 1, 26-38) ${ }^{37}$. Die Darstellung des göttlichen Ursprungs der Erleuchtung (möglicherweise spielt hierbei auch die Semantik des französischen Begriffs dé,,clar"ation eine Rolle) und des Schutzes durch das Höchste Wesen steht wiederum ganz in der Tradition der christlichen Darstellungen der vom Himmel ausgehenden oder in ihn mündenden Ereignisse der Heilsgeschichte (etwa Epiphanie-, Himmelfahrts-, Weltgerichts-, ferner Visionsbilder).

Mit Ablösung schließlich ist hier gemeint, daß eine zusammenhängende Symbolwelt durch eine andere ersetzt wird oder in einem festliegenden Bildaufbau bestimmte Figuren durch andere ersetzt werden. Eine solche Bewertung ist freilich auf viele Bilder angewiesen und kann den Status einer Wahrscheinlichkeit oder einer Vermutung nur selten ganz ablegen. In unserer Darstellung ist auf jeden Fall bemerkenswert, daß sie trotz ihrer starken „Aufladung“ mit Symbolen nur antikisierende aufnimmt, auf eindeutig christliche Symbole hingegen vollständig verzichtet. Bemerkenswert ist ferner, daß ein Porträt des Königs weggelassen ist, obschon auf das Faktum, daß die Erklärung der Menschenrechte vom König bestätigt wurde, Wert gelegt wird (s. Inschrifttafel) und sich die Allegorie der französischen Nation ungebrochen als Monarchie präsentiert. Statt des Porträts des Königs sind zwei allegorische Frauengestalten ins Bild gebracht. Folgt man der auf die Allegorisierung in der Bildwelt der Französischen Revolution generell abgestellten These von L. Hunt, könnte man hierin sogar „die Abkehr von paternalistischen oder patriarchalischen Autoritätsmodellen“ sehen, die „dem radikalen Bruch mit der Tradition und mit der Legitimation von Autorität durch Bezug auf geschichtliche Ursprünge" entsprochen habe ${ }^{38}$. In der Tat auch dies eine Ablösung - hat bei der Einführung der römischen Göttin der Freiheit als Staatsemblem durch den Konvent 1792 u. a. nachweislich der Gesichtspunkt eine Rolle gespielt, ein ähnlich zentrales Symbol der Regierung und ihrer Legitimität zu kreieren, wie es bis dahin der König gewesen war. Mit eingeflossen war aber auch die Überlegung, wie man Maria als der zentralen Frauengestalt im katholischen Bewußtsein eine adäquate Gestalt entgegenstellen könne ${ }^{39}$. Dafür gibt unser Bild selbst keinerlei Hinweise her; im Gegenteil erscheint hier eher die

37 Beschreibung und Beispiele bei J.H. Emminghaus, Art. Verkündigung an Maria, in Lexikon der Christlichen Ikonographie IV, S. 422-437.

38 Hunt, Symbole der Macht (s. Anm. 7), S. 46.

39 Vgl. ebd. S. 85, auch S. 117. - Die These scheint mir heuristisch fruchtbar und auch vertretbar, aber nicht $z$ wingend. Auch die herkömmliche christliche Ikonographie verwendete mit Vorzug weibliche Gestalten als Allegorien und Personifikation (die Tugenden, die sieben freien Künste, Ekklesia und Synagoge, auch: die göttliche Weisheit). In der profanen Kunst sollte die Popularität der Europa- und Venusmotive seit der Renaissance nicht außer Betracht gelassen werden.

\section{Copyright (c) 2004 ProQuest Information and Learning Company Copyright (c) Brill Academic Publishers}


gekrönte France als Anklang an christliche Madonnendarstellungen denn die an einen angelus interpres gemahnende szepterschwingende $\mathrm{Li}$ berté. Aber es zeigt zumindest, daß für die bildnerische Gestaltung der Botschaft eines politischen Bildes auch der Gedanke eine wichtige Rolle spielt, welche etablierten Symbole ein Äquivalent erhalten müssen, teils um die ersteren verdrängen, teils auch um von ihrer Wirkkraft profitieren zu können.

Hinsichtlich der Frage Kontinuität oder Zäsur läßt sich demnach zusammenfassend sagen: Unsere Darstellung der Menschenrechte verwendet durchgängig traditionelle Bildelemente und verschmilzt sie. Sie votiert weder für die Nichtvereinbarkeit zwischen Anerkennung der Menschenrechte und Monarchie als Staatsform, noch greift sie die christliche Tradition an oder spielt satirisch auf sie an. Andererseits enthält ihre Ausstattung mit Symbolen aber doch das deutliche Bewußtsein und auch die programmatische Ansage eines Neuanfangs. Der mit diesem Neuanfang zwangsläufig einhergehende Bruch ist aber (noch) nicht der Bruch mit der Monarchie als solcher und erst recht nicht der Durchbruch zu einer ganz neuen Ära der Menschheit im Gesamten, mit dem dann auch eine neue Zeitrechnung begänne. Vielmehr beschränkt sich der Bruch in unserer Darstellung auf die eigene nationale (französische) Vergangenheit in ihrer ständischen Formation, der Herrschaftsausübung durch eine niemand und keinem Gesetz zur Rechenschaft verpflichtete (deshalb „absolute“) Zentralmacht (der maiestas) sowie der Legitimation dieser Ordnung aus übernatürlicher Qualität. Ihr wird in antikisierenden Bildelementen und im Text die neue Ordnung auf der Grundlage der Freiheit und der gleichen Rechte jedes einzelnen gegenübergestellt, in der die Souveränität (nach H. Arendt ${ }^{40}$ ist souveraineté ursprünglich nichts anderes als die französische Übersetzung von lateinisch maiestas!) vom Volk ausgeht und die ihre Legitimation aus den mit der Vernunft erkennbaren ewigen Prinzipien bezieht, die im Menschen als solchem liegen („Natur"). Der Vergleich mit analogen Bildtypen zeigt, daß Komposition und symbolische Ausstattung kaum nur eine didaktisch-persuasive Funktion erfüllen, sondern die Erklärung der Menschen- und Bürgerrechte mit der Aura eines zu verehrenden Gegenstands umgeben - eine Intention, die ganz dem Rousseauschen Gedanken einer Zivilreligion entspräche ${ }^{41}$ und auch in den nur wenige Jahre später eingeführten republikanischen Katechismen ${ }^{42} z u$ greifen ist.

40 Arendt, Uber die Revolution (s. Anm. 30), S. 27.

41 J.-J. Rousseau, Du Contrat Social ou Principes du Droit Politique, Amsterdam 1762 , IV/8 (deutsche Ausgabe z. B. Stuttgart 1977).

42 Nähres dazu bei Hunt, Symbole der Macht (s. Anm. 7), S. 89 f.

\section{Copyright (c) 2004 ProQuest Information and Learning Company Copyright (c) Brill Academic Publishers}

